\title{
Reduced learning ability as a consequence of evolutionary adaptation to nutritional stress in Drosophila melanogaster
}

\author{
Munjong Kolss ${ }^{1}$ \& Tadeusz J. Kawecki ${ }^{1,2, *}$ \\ ${ }^{1}$ Section of Ecology and Evolution, Department of Biology, University of Fribourg, \\ Switzerland \\ ${ }^{2}$ Current address: Department of Ecology and Evolution, University of Lausanne, Le \\ Biophore, CH-1015 Lausanne, Switzerland, e-mail tadeusz.kawecki@unil.ch
}

Ecological Entomology 33, 583-588 (2008) DOI: 10.1111/j.1365-2311.2008.01007.x

Except for a few editorial changes in the proofs, this preprint is identical with the published version.

\begin{abstract}
Dietary conditions affect cognitive abilities of many species, but it is unclear to what extent this physiological effect translates into an evolutionary relationship.

2. A reduction of competitive ability under nutritional stress has been reported as a correlated response to selection for learning ability in Drosophila melanogaster. Here we test whether the reverse holds as well, i.e. whether an evolutionary adaptation to poor food conditions leads to a decrease in learning capacities.

3. Populations of D. melanogaster were (1) not subject to selection (control), (2) selected for improved learning ability, (3) selected for survival and fast development on poor food, or (4) subject to both selection regimes.

4. There was no detectable response to selection for learning ability.

5. Selection on poor food led to higher survival, faster development and smaller adult size as a direct response, and to reduced learning ability as a correlated response. This study supports the hypothesis that adaptation to poor nutrition is likely to trade off with the evolution of improved learning ability.
\end{abstract}

Keywords. Drosophila melanogaster, behaviour, learning, memory, nutritional stress, trade-off, artificial selection, experimental evolution, pleiotropy, 


\section{Introduction}

Learning ability allows an animal to adjust its behaviour in an adaptive way in novel situations (Johnston, 1982). While the benefits of learning are comparatively well understood (Dukas, 2004; Healy et al., 2005; Raine \& Chittka, 2008), we know relatively little about the associated costs. Yet, knowledge of such costs is crucial for understanding under which ecological conditions improved learning ability may evolve.

Costs of learning ability may result from allocation trade-offs. Better learning may necessitate greater allocation of energy and resources to the sensory and neural structures responsible for the acquisition, processing, storage, and retrieval of information, thus leaving less available for the maintenance of other somatic tissue, for growth, immune response, or reproduction (Dukas, 1999; Kolss et al., 2006; Laughlin, 2001; Mery \& Kawecki, 2003, 2005). At the genetic level, such trade-offs would cause alleles that improve learning to have negative pleiotropic effects on other fitness-related traits. In order for improved learning ability to evolve, the benefits must outweigh these potential costs. Moreover, the cost-benefit balance may shift depending on the environment; e.g., harsh conditions may favour other solutions to maximize fitness than favourable ones.

One ecological factor likely to impose constraints on the evolution of learning is nutritional stress. Most animal species face periods of food shortage or have to get by with food of suboptimal quality. Therefore, the ability to cope with limited nutrition is an important evolutionary adaptation. Diet quality is also likely to modulate learning ability. On the one hand, poor diet indicates that environmental conditions are hard, and learning may then be particularly useful for finding, exploring, and adapting to new food sources (Heisenberg et al., 1995). On the other hand, when food is limited, it may be unaffordable to invest much of the little available energy in the nervous system. In rodents, several studies showed that mild nutritional stress (caloric restriction) increased learning performance, particularly at old age (Dubey et al., 1996; Gould et al., 1995; Hashimoto \& Watanabe, 2005; Ingram et al., 1987; Wu et al., 2003). However, in other cases, caloric restriction had no positive effect (Bellush et al., 1996; Means et al., 1993) or even led to impaired cognitive ability, especially when applied at the prenatal or early juvenile stage (Akman et al., 2004; Lister et al., 2005; Yanai et al., 2004). In Drosophila melanogaster, flies raised at high densities had $20 \%$ more Kenyon cell fibres and a larger calyx of the mushroom body, a centre of olfactory learning in the fly brain (Heisenberg et al., 1995), but the direct effect on learning performance was not examined in that experiment. In another study, raising flies on poor-quality food reduced their performance in a visual learning task (Xia et al., 1997).

Although those findings suggest a physiological connection between diet quality and learning ability, relatively little is known about whether this translates into an evolutionary relationship as well, i.e. whether adaptation to chronic food limitation over many generations has an impact on the learning ability and vice versa. In Drosophila, a long-term selection experiment revealed that populations which had evolved improved learning performance and memory (Mery \& Kawecki, 2002) exhibited reduced ability to compete for limited food as larvae (Mery \& Kawecki, 2003). However, it has not been examined whether this trade-off is symmetric, i.e. whether populations selected for improved performance on restricted diet show decreased learning performance as a correlated response. In addition, it is conceivable that such a trade-off could be overcome 
if selection acted simultaneously on both traits. Whether this is possible depends critically on the hierarchy of resource allocation, i.e. the trade-off structure. E.g., organisms may divide energy between reproduction and somatic maintenance and then subdivide somatic resources. Genetic variation in allocation to pathways early in such hierarchies may then cause positive genetic correlations between traits that trade off later in the resource hierarchy and thus conceal this trade-off (Worley et al., 2003).

In this study, we addressed these questions by conducting a two-way factorial selection experiment. Replicate populations of $D$. melanogaster were subject to one of four regimes: (1) no selection (control), (2) selection for improved adult learning ability, (3) selection for survival and fast development on poor food, (4) selection for both improved learning ability and development on poor food. In order to avoid physiological effects of larval dietary conditions on adult learning ability, flies were only selected for each of the traits every other generation, so selection for the two traits was imposed on different individuals.

\section{Methods}

\section{Base population}

The base population was founded by mixing 200 adults from each of four Drosophila melanogaster populations. Those lines had originally been derived from a wild-type population caught in Basel, Switzerland, and kept in the lab on standard food at population sizes of about 150 adults for about 100 generations. The resulting base population was maintained on our standard cornmeal medium (15 g agar, $30 \mathrm{~g}$ sucrose, $60 \mathrm{~g}$ glucose, $12.5 \mathrm{~g}$ dry yeast, $50 \mathrm{~g}$ cornmeal, $0.5 \mathrm{~g} \mathrm{MgSO}_{4}, 0.5 \mathrm{~g} \mathrm{CaCl}_{2}, 20 \mathrm{ml}$ ethanol, $6 \mathrm{ml}$ propionic acid, and $10 \mathrm{ml}$ nipagin (10\%) per 11 of water) at a size of ca. 2000 individuals. After seven generations, the replicate selection lines were derived by collecting eggs from this base population.

\section{Selection regimes}

Four selection regimes were applied in a $2 \times 2$ (factorial) design (presence vs. absence of selection for improved learning ability $\times$ presence vs. absence of selection for survival and fast development on poor food). Preliminary experiments had suggested that adult learning ability in fruit flies may be affected by larval nutritional conditions (data not shown). Therefore, we attempted to minimize these effects by only selecting for each of the two traits in alternate generations. For logistic reasons, half of the lines of each selection regime were selected for a given trait in odd generations, the other half in even generations. Hence, there were the following four selection regimes with six replicate lines each: (1) control, i.e., subject to no selection (C-lines); (2) selection for learning ability in odd (even) generations and no selection in even (odd) generations ( $L$-lines); (3) selection for survival and fast development on poor food in even (odd) generations and no selection in odd (even) generations (P-lines); (4) selection for learning ability in odd (even) generations and selection for survival and fast development on poor food in even (odd) generations (LP-lines). 
For the selection for survival and fast development on poor food, flies were raised on a poor medium, obtained by diluting the amount of sugars, yeast, and cornmeal of the standard medium by a factor of four. Only flies which had eclosed by day 14 (usually 20$40 \%$ of the original egg number) were used to breed the next generation. Lines not selected on poor food were raised on the standard medium.

Selection for improved learning ability was carried out following the mechanical shock avoidance paradigm developed in our lab (Mery \& Kawecki, 2005; Mery et al., 2007). Conditioning and testing were done on groups of 50-70 flies (sexes mixed) on day 14 after oviposition (i.e. 3-5 days from adult eclosion). These groups were isolated by aspiration (i.e., without $\mathrm{CO}_{2}$ ) and placed in 10-ml vials $1 \mathrm{~h}$ before conditioning. They were conditioned to associate one of two odours (4-methylcyclohexanol (MCH) or 3octanol (OCT) diluted in paraffin oil $(600 \mu \mathrm{l} / \mathrm{l}))$ with mechanical shock as described in Mery \& Kawecki (2005). Two cycles of conditioning were given back to back (massed training). Three hours later, the flies were tested in complete darkness in a T-maze where they were given the choice between MCH and OCT. For each selected line, eight groups of flies (four in each direction of conditioning) were tested. Selection was carried out by taking as breeders only the flies which had made the correct choice, i.e. those which had chosen the odour previously not associated with shock.

Selection was always carried out on day 14 after oviposition (see below). Of the selected flies (or all individuals of a given line if no selection was carried out), 150 were randomly picked to breed the next generation. They were transferred to the standard medium with fresh yeast added to increase egg production and initiate re-mating before eggs for the next generation were collected seven days later. In the learning selection regime, this served to reduce the contribution of unselected males which may have mated with (some) of the selected females before selection was carried out; the other lines were then treated in the same way. All lines were thus maintained on a 21-day generation cycle at $25{ }^{\circ} \mathrm{C}$ and $70 \%$ humidity.

\section{Survival and development on poor food}

To assess the response to selection, viability (egg-to-adult survival), developmental time, and adult body mass of flies developing on the poor food were assayed for all lines. This was done after 28 generations of the experiment (i.e. 14 generations of effective selection) followed by three additional generations without selection to reduce potential differences due to maternal effects. For each line, 100 eggs were placed in each of four replicate $68-\mathrm{ml}$ vials with $10 \mathrm{ml}$ of the poor food medium and left to develop at $25{ }^{\circ} \mathrm{C}$ and $70 \%$ humidity. Each day from day 11 to 24 after oviposition, eclosed flies were removed, counted, and frozen at $-40{ }^{\circ} \mathrm{C}$. Vials were checked once more on day 28 for late individuals, the very few flies emerging on days 25-28 were pooled and treated as eclosed on day 25 . No flies emerged after day 28. The proportion of flies surviving to adulthood per vial was taken as the measure of egg-to-adult viability. In addition, the proportion of flies which eclosed by day 14 was calculated separately, as day 14 was the cut-off time in the selection on poor food (only flies emerging until then were allowed to contribute to the next generation). For each vial, the mean developmental time of all flies that emerged was calculated. 
For adult dry body mass, frozen flies from the above assay were pooled over replicate vials, sexed, dried at $80{ }^{\circ} \mathrm{C}$ for at least four days, and weighed on a Mettler MT5 balance with $1 \mu \mathrm{g}$ resolution. In general, 20 females and 20 males per line were measured; in several cases one individual was lost, and in one line five individuals were lost.

For statistical analysis, the proportion of surviving flies in each vial (both based on all emerged flies and those that eclosed within 14 days) were angularly transformed and used as response variable in an ANOVA. Using untransformed proportions did not change the results qualitatively. For developmental time, the response variable was the mean time from egg to adult eclosion calculated for each vial; using median developmental time or developmental rate (i.e. the inverse of developmental time) did not change the results qualitatively. These response variables were subject to ANOVA with learning selection regime, nutritional selection regime, and the interaction between the two as fixed factors and line nested within regime as a random factor. For developmental time, the analysis was weighted with the number of flies emerged from a given vial. For dry body mass, sex was additionally included as a fixed factor.

\section{Learning ability}

Learning ability of all lines was assayed using the same procedure as in the course of selection (i.e. the shock avoidance paradigm with two conditioning cycles and three-hour memory). This was done after 35 generations of the experiment (i.e. 17-18 generations of effective selection) followed by two generations without selection. Six replicate assays (each on a group of 50-60 flies) were performed for each line and direction of conditioning, arranged in three blocks. The assays were done in a paired fashion so that each group of flies conditioned to avoid OCT was paired with one from the same line conditioned to avoid $\mathrm{MCH}$.

The flies choosing the two arms of the T-maze were counted and the proportion of flies choosing the arm with OCT was calculated for each replicate assay. Learning scores were calculated the proportion of flies choosing OCT in a group conditioned to avoid $\mathrm{MCH}$ minus the proportion choosing OCT in the corresponding group conditioned to avoid OCT. For the analysis reported here the proportions were logit-transformed before calculating the learning scores, but the results were qualitatively the same for untransformed or angularly-transformed proportions. The learning scores were subject to an ANOVA with learning selection regime, nutritional selection regime, and the interaction between the two as fixed factors and line nested within regime and block as random factors.

\section{Olfaction controls}

In order to exclude potential differences in olfaction or unconditioned responses to odours as confounding factors, we tested odour avoidance of all lines after 35 generations (i.e. 17-18 generations of effective selection) plus two generations without selection. Groups of flies were prepared as for conditioning. They received 2 cycles of mechanical shock (with a 3-minute break in between) as during conditioning, but without any odours. 
25 minutes later, they were tested in the T-maze with one of the two odours on one side and pure paraffin oil scent on the other side. Odour avoidance was quantified as the proportion of flies moving towards the paraffin; flies staying in the central chamber of the T-maze were discarded. For each odour and each line, four tests were conducted in two blocks.

An ANOVA was performed with the logit-transformed proportions as response variable, odour, learning selection regime, nutritional selection regime, and the interaction between the two as fixed factors, and line nested within regime and block as random factors. Repeating the analysis with angularly transformed or untransformed data did not change the results qualitatively.

\section{Results}

\section{Survival and development on poor food}

At generation 28 (i.e. after 14 generations of effective selection), the lines selected on the poor food medium showed a clear increase in egg-to-adult viability (Fig. 1a; $F_{1,20}=$ $20.98, p<0.001)$. In contrast, selection for improved learning ability had no significant effect on viability $\left(F_{1,20}=0.23, p=0.64\right)$. There was no interaction between the two selection regimes $\left(F_{1,20}<0.01, p=0.98\right)$, but substantial variation among lines within regimes $\left(F_{20,72}=2.18, p<0.01\right)$. The proportion of flies eclosing within 14 days (which corresponded to the cut-off time during the selection) after oviposition showed the same pattern: selection for learning had no effect $\left(F_{1,20}<0.01, p=0.96\right)$, but selection on poor food significantly increased the proportion $\left(F_{1,20}=16.70, p<0.001\right)$. Again, the interaction between the two selection regimes was not significant $\left(F_{1,20}=3.36, p=\right.$ $0.082)$, but variation among lines was $\left(F_{20,72}=9.04, p<0.001\right)$.

Mean developmental time was significantly shorter in the lines selected on poor food (Fig. $1 \mathrm{~b} ; F_{1,20}=18.83, p<0.001$ ). Selection for improved learning ability alone had no effect on the developmental time $\left(F_{1,20}=0.10, p=0.75\right)$. However, it led to slightly shorter developmental times in the lines which were also selected on poor food (LP-lines vs. $P$-lines), while it was the opposite in the lines which were not selected on poor food (L-lines vs. $C$-lines). Thus, there was a significant interaction between the two selection regimes $\left(F_{1,20}=5.05, p=0.036\right)$. There also was significant variation among lines within regimes $\left(F_{20,72}=8.52, p<0.001\right)$.

As expected, females were heavier than males (Fig. $2 ; F_{1,916}=360.91, p<0.001$ ). In addition, selection on poor food led to significantly reduced dry body mass $\left(F_{1,20}=\right.$ $45.52, p<0.001)$, while selection for improved learning ability had no effect $\left(F_{1,20}=\right.$ $0.45, p=0.51)$. There was no interaction between the two selection regimes $\left(F_{1,20}=0.02\right.$, $p=0.89)$, but considerable variation among lines within regimes $\left(F_{20,916}=2.44, p<\right.$ $0.001)$.

\section{Learning ability}

After 35 generations (i.e. 17-18 generations of effective selection) (Fig. 3), there was no significant effect of selection for improved learning ability $\left(F_{1,20}=0.49, p=0.49\right)$ on the 
learning performance: even though the lines selected for better learning tended to do slightly better on average, the difference was small in relation to the variation among lines. However, the effect of selection on poor food was significant $\left(F_{1,20}=7.42, p=\right.$ $0.013)$ : the lines not subject to selection for development on poor food (C-lines and $L$ lines) showed learning scores around 0.22 , the selected lines (P-lines and LP-lines) only achieved scores around 0.15 , suggesting a loss of learning ability by about $30 \%$. The interaction between the two selection regimes was far from significant $\left(F_{1,20}=0.03, p=\right.$ $0.88)$, and there were no line $\left(F_{20,118}=1.26, p=0.22\right)$ or block $\left(F_{2,118}=1.01, p=0.37\right)$ effects.

\section{Olfaction controls}

Our learning test relied on the ability to perceive and respond to odours. Impaired olfaction might thus be an alternative explanation of the lower learning scores of lines selected on poor food. To exclude this confounding factor, we tested the response to odours in the absence of conditioning.

Both odours, $\mathrm{MCH}$ and OCT, were avoided, indicating that the flies could well smell them (Fig. 4). OCT was more aversive than $\mathrm{MCH}\left(F_{1,166}=20.41, p<0.001\right)$. Selection for better learning ability had no significant effect on odour avoidance $\left(F_{1,20}=\right.$ $1.44, p=0.24)$, but lines selected on poor food showed significantly stronger avoidance responses $\left(F_{1,20}=6.20, p=0.022\right)$. Thus their lower learning scores cannot be explained by impaired olfaction. Moreover, there is no significant correlation between odour avoidance and learning scores of the 24 lines (linear correlation, $r^{2}=0.06$ ); including odour avoidance as a covariate in a separate statistical analysis confirms that olfaction does not have any explanatory value for the observed learning scores (ANCOVA, $p=$ 0.87 for odour avoidance; the results for the other factors are qualitatively the same as in the ANOVA; in particular, $p=0.032$ for the larval selection regime is significant, while $p$ $=0.45$ for the learning selection regime is not). There was no significant interaction between the effects of the two selection regimes on odour avoidance $\left(F_{1,20}=0.07, p=\right.$ $0.80)$, nor a block effect $\left(F_{1,166}=0.01, p=0.92\right)$, but there was substantial variation among lines within regimes $\left(F_{20,166}=1.73, p=0.032\right)$.

\section{Discussion}

In this paper we asked if there is an evolutionary trade-off between adaptation to nutritional stress and learning ability, and whether this trade-off can be overcome by selection acting simultaneously on both traits.

In the course of experimental evolution, our $P$ and $L P$ selection lines became adapted to larval nutritional stress: they evolved higher egg-to-adult survival and faster development on poor larval food (Fig. 1). However, they paid for this adaptation with a decline in learning ability measured as $3 \mathrm{~h}$ memory in an olfactory aversive learning assay (Fig. 3). This drop in learning performance cannot be explained by deteriorated olfactory acuity; if anything, odour avoidance was somewhat stronger in the lines selected on poor food (Fig. 4). Furthermore, it is unlikely that inbreeding depression 
played a major role, considering that selection was only carried out over less than 40 generations and population size was kept moderately high (150 breeding individuals).

It is therefore more parsimonious to conclude that the loss of learning performance is presumably due to its negative genetic correlation with adaptation to poor larval food, thus indicating an evolutionary trade-off between adaptation to nutritional stress and learning ability. This fits well the previous finding where the evolution of improved learning ability in Drosophila led to reduced larval performance on restricted food (Mery \& Kawecki, 2003). Taken together, these results suggest that the two traits are linked through negative genetic pleiotropy and thus possibly through overlapping physiological or molecular mechanisms. We can only speculate on the mechanisms involved. Possibly, allometric effects may have played a role: in our selection experiment, adaptation to poor larval food was associated with shortened development (Fig. 1), resulting in a smaller adult size (Fig. 2). This may have constrained the amount of resources invested in the development or maintenance of the central nervous system or its specific components involved in learning processes, such as the mushroom bodies (Davis, 2005). Alternatively, cellular or physiological changes involved in adapting to nutritional stress may have interfered with the processes of learning acquisition, its storage in memory and/or its later retrieval. The selection lines generated in this study open the way to address the mechanisms underlying this apparent trade-off.

Unfortunately, we could not answer our second question, namely whether the trade-off between learning performance and adaptation to nutritional stress can be overcome by simultaneous selection for both traits. Our selection regime proved ineffective in improving learning performance in lines subject to selection for learning performance only ( $L$-lines; Fig. 3 )., Hence, the fact that the decline in learning ability was similar in lines which were only selected for adaptation to poor food ( $P$-lines) and those also selected for improved learning ( $L P$-lines) can also be most parsimoniously explained by selection for learning being ineffective.

The lack of response to selection for learning performance in this experiment is in contradiction with previous selection experiments on Drosophila which succeeded in producing a marked response within 15-20 generations. Mery \& Kawecki (2002) successfully selected flies for better learning in an assay, in which flies learned to associate an oviposition substrate with the aversive taste of quinine. Reif et al. (2002) obtained a response to selection on the male ability to learn to recognize unreceptive females. One could argue that while genetic variation for performance in those tasks exists, performance in the shock avoidance learning test (which was used here because it allows selection on both sexes) is a different trait which happens to show no genetic variance. However, Mery \& Kawecki's (2002) selected lines also evolved, as a correlated response, a substantially better performance in the shock avoidance assay (Mery et al., 2007). One reason why a trait may show correlated responses to selection on other traits, but little direct response, is high environmental variance, which would cause heritability of the trait to be low in spite of substantial additive genetic variation. In the shock avoidance assay, flies are selected based on a single instance of choice between two odours in a T-maze, a behaviour which is likely to contain a large random component. In contrast, in the oviposition assay used by Mery \& Kawecki (2002), selection acted on the proportion of eggs laid on the "correct" substrate during a $3 \mathrm{~h}$ period, whereby flies could 
move back and forth between the substrates. This response was thus presumably based on multiple choices done by each female, which should have reduced its random component, thus increasing heritability. Hence, large environmental variance of the response in the olfactory-shock assay may be the reason why the performance in this assay did not detectably respond to direct selection, even though it showed correlated responses to selection for performance in another learning assay (Mery \& Kawecki, 2002) and for adaptation to nutritional stress (this study). Alternatively, the response may have been countered by inadvertent selection on other traits negatively genetically correlated with the learning performance. For example, genetically "smarter" females may have had lower fecundity or "smart" males lower mating success under the conditions of the assay. In the absence of evidence for or against this possibility, the reason for the lack of direct response to selection for learning performance in this study remains in the realm of speculation.

In conclusion, our results together with those of Mery \& Kawecki (2003) point out to a symmetric evolutionary trade-off between learning ability and adaptation to nutritional stress: evolution of improved learning is correlated with reduced fitness under nutritional stress and vice versa. Such trade-offs would constrain the evolution of learning and other cognitive abilities, and thus help explain differences in those abilities within and between species (Dukas, 2004).

\section{Acknowledgements}

The authors thank L. Sygnarski and S. Rion for help with the experiments. This research was supported by the Swiss National Science Foundation and the Roche Research Foundation.

\section{References}

Akman, C., Zhao, Q., Liu, X.Z., \& Holmes, G.L. (2004) Effect of food deprivation during early development on cognition and neurogenesis in the rat. Epilepsy \& Behavior, 5, 446-454.

Bellush, L.L., Wright, A.M., Walker, J.P., Kopchick, J., \& Colvin, R.A. (1996) Caloric restriction and spatial learning in old mice. Physiology \& Behavior, 60, 541-547.

Bolhuis, J.J. (2005) Function and mechanism in neuroecology: looking for clues. Animal Biology, 55, 457-490.

Davis, R.L. (2005) Olfactory memory formation in Drosophila: From molecular to systems neuroscience. Annual Review of Neuroscience, 28, 275-302.

Dubey, A., Forster, M.J., Lal, H., \& Sohal, R.S. (1996) Effect of age and caloric intake on protein oxidation in different brain regions and on behavioral functions of the mouse. Archives of Biochemistry and Biophysics, 333, 189-197.

Dukas, R. (1999) Costs of memory: ideas and predictions. Journal of Theoretical Biology, 197, 41-50. 
Dukas, R. (2004) Evolutionary biology of animal cognition. Annual Review of Ecology Evolution and Systematics, 35, 347-374.

Gould, T.J., Bowenkamp, K.E., Larson, G., Zahniser, N.R., \& Bickford, P.C. (1995) Effects of dietary restriction on motor learning and cerebellar noradrenergic dysfunction in aged F344 rats. Brain Research, 684, 150-158.

Hashimoto, T. \& Watanabe, S. (2005) Chronic food restriction enhances memory in mice - analysis with matched drive levels. Neuroreport, 16, 1129-1133.

Healy, S.D., de Kort, S.R., \& Clayton, N.S. (2005) The hippocampus, spatial memory and food hoarding: a puzzle revisited. Trends in Ecology \& Evolution, 20, 17-22.

Heisenberg, M., Heusipp, M., \& Wanke, C. (1995) Structural plasticity in the Drosophila brain. Journal of Neuroscience, 15, 1951-1960.

Ingram, D.K., Weindruch, R., Spangler, E.L., Freeman, J.R., \& Walford, R.L. (1987) Dietary restriction benefits learning and motor-performance of aged mice. Journals of Gerontology, 42, 78-81.

Johnston, T.D. (1982) Selective costs and benefits in the evolution of learning. Advances in the Study of Behavior, 12, 65-106.

Kolss, M., Kraaijeveld, A.R., Mery, F., \& Kawecki, T.J. (2006) No trade-off between learning ability and parasitoid resistance in Drosophila melanogaster. Journal of Evolutionary Biology, 19, 1359-1363.

Laughlin, S.B. (2001) Energy as a constraint on the coding and processing of sensory information. Current Opinion in Neurobiology, 11, 475-480.

Lister, J.P., Blatt, G.J., DeBassio, W.A., Kemper, T.L., Tonkiss, J., Galler, J.R., \& Rosene, D.L. (2005) Effect of prenatal protein malnutrition on numbers of neurons in the principal cell layers of the adult rat hippocampal formation. Hippocampus, 15, 393-403.

Means, L.W., Higgins, J.L., \& Fernandez, T.J. (1993) Midlife onset of dietary restriction extends life and prolongs cognitive-functioning. Physiology \& Behavior, 54, 503508 .

Mery, F. \& Kawecki, T.J. (2002) Experimental evolution of learning ability in fruit flies. Proceedings of the National Academy of Sciences of the United States of America, 99, 14274-14279.

Mery, F. \& Kawecki, T.J. (2003) A fitness cost of learning ability in Drosophila melanogaster. Proceedings of the Royal Society of London Series B-Biological Sciences, 270, 2465-2469.

Mery, F. \& Kawecki, T.J. (2005) A cost of long-term memory in Drosophila. Science, 308, 1148-1148.

Mery, F., Pont, J., Preat, T., \& Kawecki, T.J. (2007) Experimental evolution of olfactory memory in Drosophila melanogaster. Physiological and Biochemical Zoology, 80, $399-405$. 
Raine, N.E. \& Chittka, L. (2008) The correlation of learning speed and natural foraging success in bumble-bees. Proceedings of the Royal Society B-Biological Sciences.

Reif, M., Linsenmair, K.E., \& Heisenberg, M. (2002) Evolutionary significance of courtship conditioning in Drosophila melanogaster. Animal Behaviour, 63, 143-155.

Worley, A.C., Houle, D., \& Barrett, S.C.H. (2003) Consequences of hierarchical allocation for the evolution of life-history traits. American Naturalist, 161, 153-167.

Wu, A.G., Sun, X.F., \& Liu, Y.G. (2003) Effects of caloric restriction on cognition and behavior in developing mice. Neuroscience Letters, 339, 166-168.

Xia, S.Z., Liu, L., Feng, C.H., \& Guo, A.K. (1997) Nutritional effects on operant visual learning in Drosophila melanogaster. Physiology \& Behavior, 62, 263-271.

Yanai, S., Okaichi, Y., \& Okaichi, H. (2004) Long-term dietary restriction causes negative effects on cognitive functions in rats. Neurobiology of Aging, 25, 325-332. 

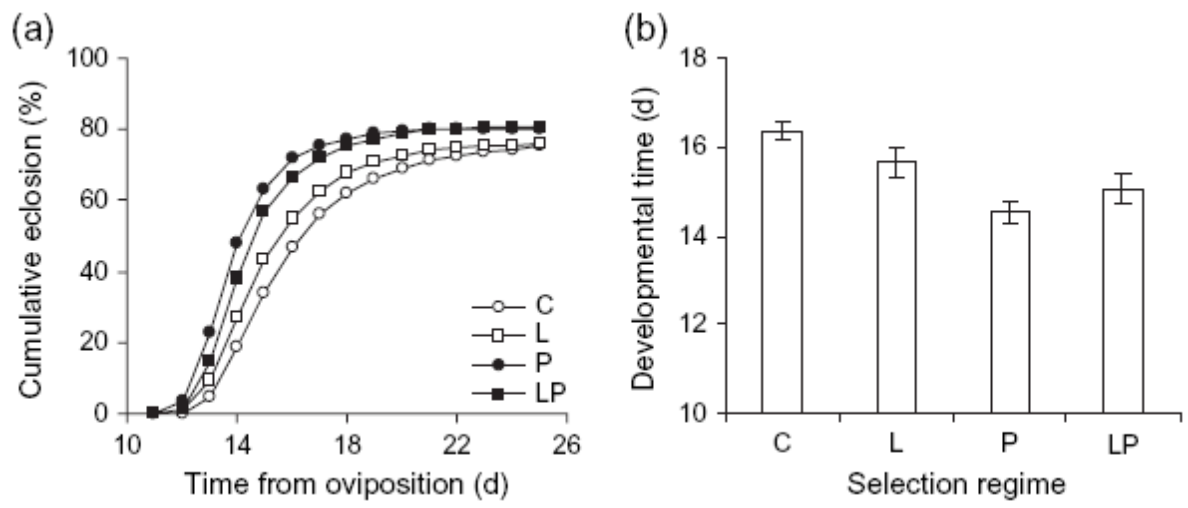

Fig. 1: Cumulative emergence curve (a) and developmental time (b) of flies from the four selection regimes raised on the poor food medium (means \pm S.E.). Six lines each were selected for improved learning ability only (L-lines), selected for survival and fast development on poor food only ( $P$-lines), selected for both (LP-lines), or unselected controls ( $C$-lines). For the sake of readability, standard error bars were not plotted in panel (a). Of the 60 points plotted only four had standard error greater than $5 \%$; for overall egg-to-adult survival (i.e., cumulative eclosion until day 25) the standard errors are all smaller than $1.5 \%$.

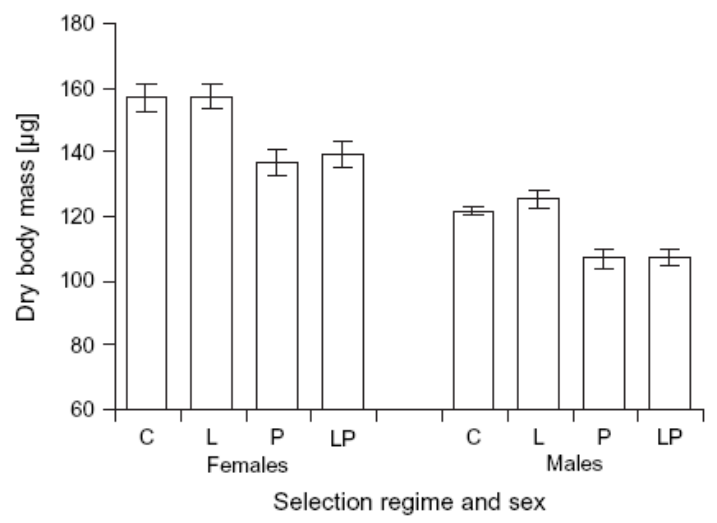

Fig. 2: Adult dry body mass (means \pm S.E.) of flies from the four selection regimes grown on the poor food medium. 


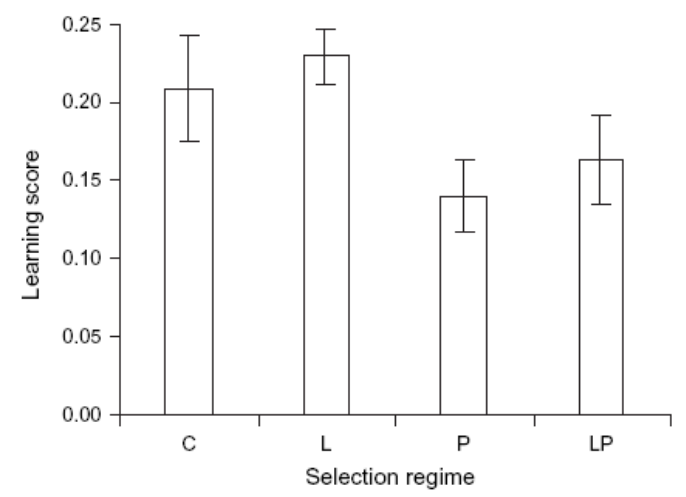

Fig. 3: Three-hour memory (means \pm S.E.) of flies from the four selection regimes (grown on normal food).

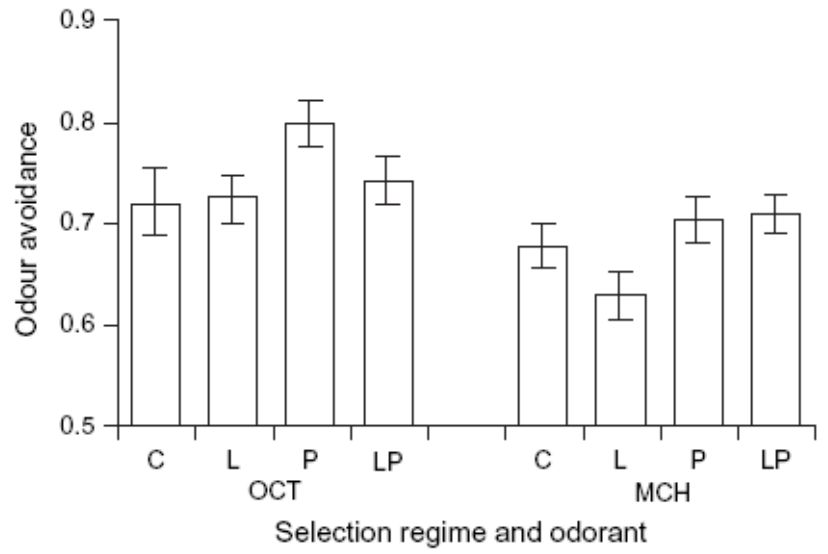

Fig. 4: Avoidance (means \pm S.E.) of the two odorants 3-octanol (OCT) and 4methylcyclohexanol $(\mathrm{MCH})$, quantified as the proportion of flies choosing pure paraffin vs. the respective odorant dissolved in paraffin. 